\title{
On the irreducibility of multivariate subresultants
}

\section{Sur l'irréductibilité des sous-résultants multivariés}

\author{
Laurent Busé ${ }^{a}$ Carlos D' Andrea ${ }^{b}$ \\ ${ }^{\mathrm{a}}$ INRIA, GALAAD, 2004 route des Lucioles, B.P. 93, 06902 Cedex France. \\ ${ }^{\mathrm{b}}$ Department of Mathematics UC Berkeley \&3 The Miller Institute for Basic Research in Science. 1089 Evans Hall \\ Berkeley, CA 94720-3840 USA
}

\begin{abstract}
Let $P_{1}, \ldots, P_{n}$ be generic homogeneous polynomials in $n$ variables of degrees $d_{1}, \ldots, d_{n}$ respectively. We prove that if $\nu$ is an integer satisfying $\sum_{i=1}^{n} d_{i}-n+1-\min \left\{d_{i}\right\}<\nu$, then all multivariate subresultants associated to the family $P_{1}, \ldots, P_{n}$ in degree $\nu$ are irreducible. We show that the lower bound is sharp. As a byproduct, we get a formula for computing the residual resultant of $\left(\begin{array}{c}\rho-\nu+n-1 \\ n-1\end{array}\right)$ smooth isolated points in $\mathbb{P}^{n-1}$.

\section{Résumé}

Soient $P_{1}, \ldots, P_{n}$ des polynômes homogènes génériques en $n$ variables de degré respectif $d_{1}, \ldots, d_{n}$. Nous montrons que si $\nu$ est un entier tel que $\sum_{i=1}^{n} d_{i}-n+1-\min \left\{d_{i}\right\}<\nu$, tous les sous-résultants multivariés de degré $\nu$ des polynômes $P_{1}, \ldots, P_{n}$ sont irréductibles. Nous montrons également que cette borne est atteinte dans des cas particuliers. Comme conséquence directe nous obtenons une nouvelle formule pour le calcul du résultant résiduel de $\left(\begin{array}{c}\rho-\nu+n-1 \\ n-1\end{array}\right)$ points lisses isolés dans $\mathbb{P}^{n-1}$.
\end{abstract}

Classical subresultants of two univariate polynomials have been studied by Sylvester in the foundational work [13]. Multivariate subresultants, introduced in [2], provide a criterion for over-constrained polynomial systems to have Hilbert function of prescribed value, generalizing the classical case. To be more precise, let $\mathbb{K}$ be a field. If $P_{1}, \ldots, P_{s}$ are homogeneous polynomials in $\mathbb{K}\left[X_{1}, \ldots, X_{n}\right]$ with $d_{i}=\operatorname{deg}\left(P_{i}\right)$ and $s \leq n$, $H_{d_{1}, \ldots, d_{s}}($.$) is the Hilbert function of a complete intersection given by s$ homogeneous polynomials in $n$ variables of degrees $d_{1}, \ldots, d_{s}$, and $S$ is a set of $H_{d_{1}, \ldots, d_{s}}(\nu)$ monomials of degree $\nu$, the subresultant $\Delta_{S}^{\nu}$ is a polynomial in the coefficients of the $P_{i}^{\prime} s$ of degree $H_{d_{1}, \ldots, d_{i-1}, d_{i+1}, \ldots, d_{n}}\left(\nu-d_{i}\right)$ in the coefficients of $P_{i}$ $(i=1, \ldots, s)$ having the following universal property: $\Delta_{S}^{\nu} \neq 0$ if and only if $I_{\nu}+\mathbb{K}\langle S\rangle=\mathbb{K}\left[X_{1}, \ldots, X_{n}\right]_{\nu}$, where $I_{\nu}$ is the degree $\nu$ part of the ideal generated by the $P_{i}$ 's (see [2]).

Multivariate subresultants have been used in computational algebra for polynomial system solving ([10],[14]) as well as for providing explicit formulas for the representation of rational functions $([11,6,7,12])$.

Email addresses: lbuse@sophia.inria.fr (Laurent Busé), cdandrea@math.berkeley.edu (Carlos D'Andrea). 
The study of their properties is an active area of research $([3,4,6,7,8])$. In particular, it is important to know which $S$ verify $\Delta_{S}^{\nu} \neq 0$, and which of these $\Delta_{S}^{\nu}$ are irreducible (see the final remarks and open questions in [2] and the conjectures in [7]). Partial results have been obtained in this direction. In [5] it is shown that, if $s=n$ and $\sum_{i=1}^{n} d_{i}-n-\min \left\{d_{i}\right\}<\nu$, then for every set $S$ of monomials of degree $\nu$ and cardinal $H_{d_{1}, \ldots, d_{n}}(\nu)$, the polynomial $\Delta_{S}^{\nu}$ is not identically zero. Moreover, in [4], it is also proven that if $s=n, \nu=\sum_{i=1}^{n} d_{i}-n$, and $S=\left\{x_{j}^{\nu}\right\}$ for $j=1, \ldots, n$, then $\Delta_{S}^{\nu}$ is an irreducible polynomial in the coefficients of the $P_{i}^{\prime} s$. In [8, Lemma 4.2] the irreducibility of $\Delta_{S}^{\nu}$ is shown for $s=n=2, \max \left\{d_{1}, d_{2}\right\} \leq \nu$, and $S=\left\{X_{2}^{\nu}, X_{1} X_{2}^{\nu-1}, \ldots, X_{1}^{H_{d_{1}, d_{2}}(\nu)-1} X_{2}^{\nu-H_{d_{1}, d_{2}}(\nu)+1}\right\}$.

In this note we study the irreducibility problem in the case $s=n$. Let us introduce some notations in order to state our result. Let $\rho:=\sum_{i=1}^{n}\left(d_{i}-1\right)$. For $i=1, \ldots, n$ and $\alpha \in \mathbb{Z}_{\geq 0}^{n}$ such that $|\alpha|=d_{i}$, introduce a new variable $c_{i, \alpha}$. Let $\mathbb{A}:=\mathbb{Z}\left[c_{i, \alpha}, i=1, \ldots, n,|\alpha|=d_{i}\right]$ and set

$$
P_{i}\left(x_{1}, \ldots, x_{n}\right):=\sum_{|\alpha|=d_{i}} c_{i, \alpha} x^{\alpha} .
$$

Theorem For every $\nu$ such that $\rho-\min \left\{d_{i}\right\}+1<\nu$ and every set $S$ of monomials of degree $\nu$ and cardinality $H_{d_{1}, \ldots, d_{n}}(\nu)$, the subresultant $\Delta_{S}^{\nu}\left(P_{1}, \ldots, P_{n}\right)$ is irreducible in $\mathbb{A}$.

Observe that, if $n=2$, then $\rho-\min \left\{d_{i}\right\}+1=d_{1}+d_{2}-2-\min \left\{d_{i}\right\}+1=\max \left\{d_{i}\right\}-1$, and this is equivalent to $\max \left\{d_{i}\right\} \leq \nu$, so our result contains those in [8].

Proof of the Theorem: For simplicity we assume hereafter that $d_{1} \geq \ldots \geq d_{n} \geq 1$. First observe that if $\nu>\rho$ then $\Delta_{S}^{\nu}$ is simply a resultant, and is hence known to be irreducible. So, we can suppose w.l.o.g. that $d_{n}>1$. We thus only have to consider integers $\nu$ such that

$$
\rho \geq \nu>\rho-d_{n}+1=\sum_{i=1}^{n-1}\left(d_{i}-1\right),
$$

where we recall that $\rho=\sum_{i=1}^{n}\left(d_{i}-1\right)$. We begin by computing the multi-degree of the subresultants $\Delta_{S}^{\nu}$; we know (see [2]) that

$$
\operatorname{deg}_{P_{i}}\left(\Delta_{S}^{\nu}\right)=H_{d_{1}, \ldots, d_{i-1}, d_{i+1}, \ldots, d_{n}}\left(\nu-d_{i}\right) .
$$

But from the standard short exact sequence

$$
0 \rightarrow \frac{R}{\left(f_{1}, \ldots, f_{i-1}, f_{i+1}, \ldots, f_{n}\right)}\left(-d_{i}\right) \stackrel{\times f_{i}}{\longrightarrow} \frac{R}{\left(f_{1}, \ldots, f_{i-1}, f_{i+1}, \ldots, f_{n-1}\right)} \rightarrow \frac{R}{\left(f_{1}, \ldots, f_{n}\right)} \rightarrow 0,
$$

where $f_{1}, \ldots, f_{n}$ are homogeneous polynomials of respective degree $d_{i}$ in a graded polynomial ring $R$ and $f_{1}, \ldots, f_{n}$ is a complete intersection in $R$, we deduce

$$
H_{d_{1}, \ldots, d_{i-1}, d_{i+1}, \ldots, d_{n}}\left(t-d_{i}\right)=H_{d_{1}, \ldots, d_{i-1}, d_{i+1}, \ldots, d_{n}}(t)-H_{d_{1}, \ldots, d_{n}}(t),
$$

for all integer $t$. It follows that for all integer $\nu \geq \rho-d_{n}+1$,

$$
\operatorname{deg}_{P_{i}}\left(\Delta_{S}^{\nu}\right)=\frac{d_{1} \ldots d_{n}}{d_{i}}-H_{d_{1}, \ldots, d_{n}}(\nu)=\frac{d_{1} \ldots d_{n}}{d_{i}}-\left(\begin{array}{c}
\rho-\nu+n-1 \\
n-1
\end{array}\right),
$$

where that last equality comes from the facts that $H_{d_{1}, \ldots, d_{n}}(\rho-t)=H_{d_{1}, \ldots, d_{n}}(t)$ for all integer $t$, and $H_{d_{1}, \ldots, d_{n}}(t)=\left(\begin{array}{c}t+n-1 \\ n-1\end{array}\right)$ for all $0 \leq t<d_{n}$. We define $\mathbf{a}:=\left(\begin{array}{c}\rho-\nu+n-1 \\ n-1\end{array}\right)$. As a does not depend on $i \in$ $\{1, \ldots, n\}$ and residual (or reduced) resultants of a isolated points in $\mathbb{P}^{n-1}$ have the same degree in the coefficients of $P_{i}$ as the right hand side of (3), this suggest that we compare $\Delta_{S}^{\nu}$ with residual resultants.

We will work with an ideal $G$ defining a points in $\mathbb{P}^{n-1}$ which is generated in degree at most $d_{n}$ and such that $G_{d_{n}-1} \neq 0$. Ideals defining a points in sufficiently generic position are generated in degree exactly $\rho-\nu+1$ (see [9, Proposition 4]). Since by (2) we have $d_{n}>\rho-\nu+1$, we thus choose such an 
ideal $G=\left(g_{1}, \ldots, g_{m}\right)$, where $\operatorname{deg}\left(g_{i}\right)=\rho-\nu+1$ for all $i=1, \ldots, m$, defining a points in generic position (see [9] for the definition of "generic position"), and hence locally a complete intersection.

Now consider the following specialization of polynomials $P_{i}$ 's

$$
P_{i} \mapsto \bar{P}_{i}:=\sum_{j=1}^{m} p_{i j}(x) g_{j}(x),
$$

where $p_{i j}(x)=\sum_{|\alpha|=d_{i}-\rho+\nu-1} c_{i j}^{|\alpha|} x^{\alpha}$ is a generic polynomial of degree $d_{i}-\rho+\nu-1$. There exists a resultant associated to the system $\bar{P}_{1}, \ldots, \bar{P}_{n}$, called the residual resultant. We denote it by $\operatorname{Res}_{G}\left(\bar{P}_{1}, \ldots, \bar{P}_{n}\right)$. Let us recall its main properties (see [1] $\S 3.1$ ).

- $\operatorname{Res}_{G}\left(\bar{P}_{1}, \ldots, \bar{P}_{n}\right)$ is a homogeneous and irreducible polynomial in the ring of all the coefficients $\mathbb{Q}\left[c_{i j}^{|\alpha|}\right]$,

- For any given specialization of the coefficients $c_{i j}^{|\alpha|}$, s sending $\bar{P}_{i}$ to $Q_{i}$, we have

$$
\operatorname{Res}_{G}\left(Q_{1}, \ldots, Q_{n}\right)=0 \Longleftrightarrow\left(Q_{1}, \ldots, Q_{n}\right)^{\text {sat }} \varsubsetneqq G=G^{\text {sat }},
$$

- $\operatorname{Res}_{G}\left(\bar{P}_{1}, \ldots, \bar{P}_{n}\right)$ is multi-homogeneous: it is homogeneous in the coefficients of each polynomials $\bar{P}_{i}$, $i=1, \ldots, n$, and we have

$$
\operatorname{deg}_{\bar{P}_{i}}\left(\operatorname{Res}_{G}\left(\bar{P}_{1}, \ldots, \bar{P}_{n}\right)\right)=\frac{d_{1} \ldots d_{n}}{d_{i}}-\mathbf{a} .
$$

We are now going to compare this residual resultant with the specialized subresultant $\Delta_{S}^{\nu}\left(\bar{P}_{1}, \ldots, \bar{P}_{n}\right)$, which is non-zero as proved in [4]. We claim that we have the following implications:

$$
\Delta_{S}^{\nu}\left(Q_{1}, \ldots, Q_{n}\right) \neq 0 \Rightarrow H_{(\underline{Q})}(\nu)=\mathbf{a} \Rightarrow H_{(\underline{Q})}(t)=\mathbf{a} \forall t \geq \nu \Rightarrow \operatorname{Res}_{G}\left(Q_{1}, \ldots, Q_{n}\right) \neq 0,
$$

where $H_{(Q)}($.$) denotes the Hilbert function associated to the ideal \left(Q_{1}, \ldots, Q_{n}\right)$. Only the second implication needs to be proved, the others follow directly from the algebraic properties of resultants and subresultants. We know that $H_{G}(t)=\mathbf{a}$ for all $t \geq \rho-\nu+1$ (see [9]), and since we have supposed (2), it is a straightforward computation to show that $\nu \geq \rho-\nu+1$. By hypothesis $(\underline{Q}) \subset G$ and $\operatorname{dim}_{\mathbb{K}}(\underline{Q})_{\nu}=\operatorname{dim}_{\mathbb{K}} G_{\nu}$, so we can choose a basis $e_{1}, \ldots, e_{l}$ of both $\mathbb{K}$-vector spaces $(\underline{Q})_{\nu}$ and $\overline{G_{\nu}}$, where $e_{i} \in(\underline{Q})_{\nu}$. But

$\operatorname{dim}_{\mathbb{K}} G_{\nu+1}=\operatorname{dim}_{\mathbb{K}}\left(\mathbb{K}\left[x_{1}, \ldots, x_{n}\right]_{\nu+1}\right)-\mathbf{a}=\operatorname{dim}_{\mathbb{K}}\left(x_{1} G_{\nu}+x_{2} G_{\nu}+\cdots+x_{n} G_{\nu}\right)=\operatorname{dim}_{\mathbb{K}} \sum_{i=1}^{n} x_{i}\left\langle e_{1}, \ldots, e_{l}\right\rangle_{\mathbb{K}}$,

and since $e_{i} \in(\underline{Q})$ it follows $\operatorname{dim}_{\mathbb{K}}(\underline{Q})_{\nu+1} \geq \operatorname{dim}_{\mathbb{K}}\left(\mathbb{K}\left[x_{1}, \ldots, x_{n}\right]_{\nu+1}\right)-\mathbf{a}$. As $(\underline{Q}) \subset G$ this inequality is an equality and hence $H_{(\underline{Q})}(\nu+1)=\overline{\mathbf{a}}$. By induction we obtain our claimed implication.

Due to $(5)$ and the irreducibility of the residual resultant, we deduce that $\operatorname{Res}_{G}\left(\bar{P}_{1}, \ldots, \bar{P}_{n}\right)$ divides $\Delta_{S}^{\nu}\left(\bar{P}_{1}, \ldots, \bar{P}_{n}\right)$. But both polynomials have the same degree, so they must be equal up to a rational number (giving a new formula for computing this residual resultant using [3]). Since this residual resultant is irreducible, and since $\Delta_{S}^{\nu}$ and $\Delta_{S}^{\nu}\left(\bar{P}_{1}, \ldots, \bar{P}_{n}\right)$ have the same multi-degree, this shows that $\Delta_{S}^{\nu}$ is irreducible in $\mathbb{Q}\left[\operatorname{coeff}\left(P_{i}\right)\right]$.

It remains to prove that $\Delta_{S}^{\nu}$ is irreducible in $\mathbb{Z}\left[\operatorname{coeff}\left(P_{i}\right)\right]$. As it is irreducible in $\mathbb{Q}\left[\operatorname{coeff}\left(P_{i}\right)\right]$, we only have to show that $\Delta_{S}^{\nu}$ has content \pm 1 . Suppose that this is not the case, and let $p \in \mathbb{Z}$ be a prime dividing the content of $\Delta_{S}^{\nu}$. Let $k$ be the algebraic closure of $\mathbb{Z}_{p}$. This implies that $\Delta_{S}^{\nu}=0$ in $K:=k\left(\operatorname{coeff}\left(P_{i}\right)\right)$, and hence $S$ is linearly dependent in $K\left[x_{1}, \ldots, x_{n}\right] /\left\langle P_{1}, \ldots, P_{n}\right\rangle$, contradicting the main result of [4].

Reducibility in lower degrees: We now exhibit some sets $S$ of degree $\nu=\rho-\min \left\{d_{i}\right\}+1$ such that $\Delta_{S}^{\nu}$ factorizes. This shows that the lower bound in our theorem is sharp. 
- $\mathbf{n}=\mathbf{2}, \mathbf{d}_{\mathbf{1}}>\mathbf{d}_{\mathbf{2}}$ : In this case, $\nu=d_{1}-1 \geq d_{2}$, and $H_{d_{1}, d_{2}}(\nu)=d_{2}$. If we set $f_{2}=c_{0} x_{1}^{d_{2}}+c_{1} x_{1}^{d_{2}-1} x_{2}+$ $\ldots+c_{d_{2}} x_{2}^{d_{2}}$, and choose $S:=\left\{x_{1}^{d_{1}-d_{2}} x_{2}^{d_{2}-1}, x_{1}^{d_{1}-d_{2}+1} x_{2}^{d_{2}-2}, \ldots, x_{1}^{d_{1}-1}\right\}$. Thus $\Delta_{S}^{\nu}$ is the determinant of the following upper triangular Sylvester type matrix of order $d_{1}-d_{2}+1$ :

$$
\left(\begin{array}{cccccccc}
c_{0} & c_{1} & \cdots & c_{d_{2}} & 0 & 0 & \cdots & 0 \\
0 & c_{0} & c_{1} & \ldots & c_{d_{2}} & 0 & \ldots & 0 \\
0 & 0 & c_{0} & c_{1} & \cdots & c_{d_{2}} & 0 & \vdots \\
0 & 0 & 0 & \ddots & \vdots & \vdots & \vdots & \vdots \\
0 & 0 & 0 & 0 & \ddots & \vdots & \vdots & \vdots \\
0 & 0 & 0 & 0 & 0 & c_{0} & c_{1} & c_{2} \\
0 & 0 & 0 & 0 & 0 & 0 & c_{0} & c_{1} \\
0 & 0 & 0 & 0 & 0 & 0 & 0 & c_{0}
\end{array}\right),
$$

so it cannot be irreducible if $d_{1}>d_{2}$.

- $\mathbf{n}>\mathbf{2}, \mathbf{d}_{\mathbf{1}}-\mathbf{1}>\mathbf{d}_{\mathbf{2}}=\mathbf{d}_{\mathbf{3}}=\ldots=\mathbf{d}_{\mathbf{n}}=\mathbf{1}$ : Again in this case, $\nu=d_{1}-1$ and $H_{d_{1}, d_{2}}(\nu)=1$. Choose $S=\left\{x_{1}^{\nu}\right\}$ and, if $f_{i}=c_{1 i} x_{1}+\ldots+c_{n i} x_{n}, i=2, \ldots, n$, we set $\delta:=\operatorname{det}\left(c_{i j}\right)_{2 \leq i, j \leq n}$. Applying Lemma 4.4 in [6] to this situation, we get that $\Delta_{S}^{\nu}=\delta^{\nu}$. So, $\Delta_{S}^{\nu}$ is not irreducible.

Acknowledgements: This work started during the special year (2002-2003) on "Commutative Algebra" at the Mathematical Sciences Research Institute (MSRI Berkeley). We wish to thank MSRI for its hospitality and for the wonderful working atmosphere it provided. We are also grateful to David Cox, Gabriela Jeronimo and Bernd Sturmfels for their helpful comments on preliminary versions of this paper.

\section{References}

[1] Busé, L. Étude du résultant sur une variété algébrique. Ph.D. Thesis, Université de Nice, 2001.

[2] Chardin, Marc. Multivariate subresultants. J. Pure Appl. Algebra 101 (1995), no. 2, 129-138.

[3] Chardin, Marc. Formules à la Macaulay pour les sous-résultants en plusieurs variables. C. R. Acad. Sci. Paris Sér. I Math. 319 (1994), no. 5, 433-436.

[4] Chardin, Marc. Formules à la Macaulay pour les sous-résultants en plusieurs variables et application au calcul d'un résultant réduit. Preprint (extended version of the previous article). Available at http://www.math.jussieu.fr/ chardin/textes.html

[5] Chardin, Marc. Sur l'indépendance linéaire de certains monômes modulo des polynômes génériques. C. R. Acad. Sci. Paris Sér. I Math. 319 (1994), no. 10, 1033-1036.

[6] D'Andrea, Carlos; Jeronimo, Gabriela. Subresultants and Generic Monomial bases. Preprint 2003.

[7] D'Andrea, Carlos; Khetan, Amit. Macaulay style formulas for toric residues. Preprint 2003.

[8] El Kahoui, M'hammed. An elementary approach to subresultants theory. J. Symbolic Comput. 35 (2003), no. 3, 281-292.

[9] Geramita, A. V.; Orecchia, F. Minimally generating ideals defining certain tangent cones. J. Algebra 78 (1982), no. 1, $36-57$.

[10] González-Vega, Laureano. Determinantal formulae for the solution set of zero-dimensional ideals. J. Pure Appl. Algebra 76 (1991), no. 1, 57-80.

[11] Jouanolou, J. P. Formes d'inertie et résultant: un formulaire. Adv. Math. 126 (1997), no. 2, 119-250.

[12] Mulders, Thom. A note on subresultants and the Lazard/Rioboo/Trager formula in rational function integration. J. Symbolic Comput. 24 (1997), no. 1, 45-50.

[13] Sylvester, Joseph H. A Theory of syzygetic relations of two rational integral functions, comprising an application to the theory of sturm's functions, and that of the greatest algebraic common measure. Philosophical Transl, 143 (1853) $407-548$. 
[14] Szanto, Agnes. Solving over-determined systems by subresultant methods. J. Symbolic Comput. To appear. 